\title{
ON DIFFERENTIABLY SIMPLE ALGEBRAS $\left({ }^{1}\right)$
}

BY

LAURENCE R. HARPER, JR.

1. Introduction. It is known (see Albert [1]) that every simple commutative power-associative algebra of degree $t>2$ over an algebraically closed field $\mathfrak{F}$ of characteristic $p>5$ is a Jordan algebra. Moreover, in the partially stable case, a characterization of the simple algebras of degree two is given by Albert in [3]. In his theory Albert expresses the structure of simple partially stable algebras in terms of certain commutative associative algebras $\mathfrak{B}$ over $\mathfrak{F}$. These commutative associative algebras have unity elements, and each algebra $\mathfrak{B}$ is differentiably simple relative to some set of derivations of $\mathfrak{B}$ over $\mathfrak{F}$. In this paper we shall determine the structure of the algebras $\mathfrak{B}$ and derive a property of simple partially stable algebras which follows from Albert's characterization.

Let $\mathfrak{B}$ be a commutative associative algebra with unity element $e$ over $\mathfrak{F}$. We shall now define a commutative power-associative algebra $\mathfrak{I}$ over $\mathfrak{F}$ which is the essential subalgebra of a partially stable commutative powerassociative algebra $\subseteq$ as defined by Albert in [3]. Let $m \geqq 2$ and let $y_{i} \mathscr{B}$ denote a homomorphic image of the vector space $\mathfrak{B}$ for $i=0, \cdots, m$. Then $\mathfrak{I}$ will be the vector space direct sum

$$
\mathfrak{T}=\mathfrak{B}+\mathfrak{R}
$$

where $\mathbb{R}$ is the sum, not necessarily direct, of the component spaces $y_{0} \mathfrak{B}, \cdots$, $y_{m} \mathfrak{B}$. Select elements $b_{i j}$ in $\mathfrak{B}$ and derivations $D_{i j}$ of $\mathfrak{B}$ over $\mathfrak{F}$ such that

$$
\begin{array}{ll}
b_{i j} & =b_{j i}, \quad b_{00}=e, \quad b_{0 j}=0 \\
D_{i j} & =-D_{j \imath}
\end{array}
$$

for $i, j=0, \cdots, m$ where then $D_{i i}=0$ for $i=0, \cdots, m$. We now define products in $\mathfrak{T}$ by assuming that $\mathfrak{B}$ is a subalgebra of $\mathfrak{T}$, that

$$
\left(y_{i} a\right) b=y_{i}(a b)=b\left(y_{i} a\right)
$$$$
(i=0, \cdots, m)
$$

for all elements $a$ and $b$ of $\mathfrak{B}$, and finally that

$$
\left(y_{i} a\right)\left(y_{j} b\right)=b_{i j} a b+\left(a D_{i j}\right) b-a\left(b D_{i j}\right)
$$

for all $a$ and $b$ of $\mathfrak{B}$ and $i, j=0, \cdots, m$. The result will be a commutative power-associative algebra of degree two over $\mathfrak{F}$.

Received by the editors July $9,1960$.

(1) This paper is essentially the author's Ph.D. thesis at the University of Chicago. Sincere thanks are due Professor A. A. Albert for his encouragement and many helpful suggestions. 
We shall also require that the $b_{i j}$ and the $D_{i j}$ be chosen so that:

The algebra $\mathfrak{B}$ is $\left\{D_{i j}\right\}$-simple.

If $g$ is in $\mathfrak{l}$ and $g u=0$ for all $u$ in $\mathfrak{l}$, then $g=0$.

It is one of the principal results of Albert in [3] that these conditions are equivalent to the simplicity of the partially stable algebra $\mathfrak{S}$ mentioned above. It is known [2] that condition (A) implies that

$$
\mathfrak{B}=e \mathfrak{F}+\mathfrak{R}
$$

where $\mathfrak{N}$ is the radical of $\mathfrak{B}$ and $x^{p}=0$ for every element $x$ in $\mathfrak{N}$. We shall completely determine the structure of $\mathfrak{B}$, and we state our main result as

THEOREM 1. Let $\mathfrak{B}$ be a commutative associative algebra with unity e over an algebraically closed field $\mathfrak{F}$, and let $\mathfrak{B}$ be differentiably simple relative to a set of derivations of $\mathfrak{B}$ over $\mathfrak{F}$. Then $\mathfrak{B}=\mathfrak{F}\left[e, x_{1}, \cdots, x_{n}\right]$ is an algebra with generators $x_{1}, \cdots, x_{n}$ over $\mathfrak{F}$ which are independent except for the relations $x_{1}^{p}=\ldots$ $=x_{n}^{p}=0$ where $p>0$ is the characteristic of $\mathfrak{F}$.

In all examples of the algebras $\mathfrak{T}$ given to date the space $\mathfrak{R}$ has been a direct sum of the components $y_{0} \mathfrak{B}, \cdots, y_{m} \mathfrak{B}$. As our final result we shall construct a class of examples of the algebras $\mathfrak{I}$ in which $\mathfrak{l}=\left(y_{0} \mathfrak{B}, \cdots, y_{m} \mathfrak{B}\right)$ with $m=2$ and $\mathfrak{R}$ is not a direct sum and cannot be represented as a direct sum in this manner.

2. The algebra $\mathfrak{B}$. Let $\mathfrak{B}$ be a commutative associative algebra with unity element $\bar{e}$ over $\mathfrak{F}$, and let $\mathfrak{B}$ be $\overline{\mathfrak{D}}$-simple for some set $\overline{\mathfrak{D}}$ of derivations of $\mathfrak{B}$ over $\mathfrak{F}$. Then by (6) we may write

$$
\mathfrak{B}=\bar{e} \mathfrak{F}+\overline{\mathfrak{R}}
$$

where $x^{p}=0$ for each $x$ in $\overline{\mathfrak{R}}$. The algebra $\mathfrak{B}$, being finite dimensional, is finitely generated. Let $\left\{\bar{e}, \bar{x}_{1}, \cdots, \bar{x}_{n}\right\}$ be a set of generators of $\mathfrak{B}$ which is minimal in the sense that no set containing $\bar{e}$ and having fewer elements generates $\mathfrak{B}$. Also let

$$
\mathfrak{A}=\mathfrak{F}\left[e, x_{1}, \cdots, x_{n}\right]
$$

be the commutative associative algebra generated over $\mathfrak{F}$ by generators $e, x_{1}, \cdots, x_{n}$ which are independent except for the relations $e^{2}=e, e x_{i}=x_{i}$, and $x_{i}^{p}=0$ which hold for $i=1, \cdots, n$. It is clear that the mappings $e \rightarrow \bar{e}$, $x_{i} \rightarrow \bar{x}_{i}(i=1, \cdots, n)$ define a homomorphism $\phi$ of $\mathfrak{A}$ onto $\mathfrak{B}$. We let $\mathfrak{M}$ be the kernel of $\phi$. We see that Theorem 1 will be proved if we can show that $\mathfrak{M}=0$.

We now note some properties of $\mathfrak{A}$. We may write

$$
\mathfrak{A}=e \mathfrak{F}+\mathfrak{N}
$$


where $\mathfrak{N}=\mathfrak{F}\left[x_{1}, \cdots, x_{n}\right]$ is the radical of $\mathfrak{A}$ and consists of all polynomials in the $x_{i}$ with constant term zero. We observe that every element of $\mathfrak{A}$ which is not in $\mathfrak{N}$ has an inverse. For if $a=\alpha+u$ with $\alpha$ in $\mathfrak{F}, u$ in $\mathfrak{N}, \alpha \neq 0$, then $a^{-1}=\left(\alpha^{p}\right)^{-1}(\alpha+u)^{p-1}$. Also it is known [4] that the derivation algebra of $\mathfrak{A}$ consists of all linear transformations $D=D\left(a_{1}, \cdots, a_{n}\right)$ of $\mathfrak{A}$ defined by

$$
a D=\left(\partial a / \partial x_{1}\right) a_{1}+\cdots+\left(\partial a / \partial x_{n}\right) a_{n}
$$

where $a_{1}, \cdots, a_{n}$ are in $\mathfrak{A}$ and $\partial a / \partial x_{i}$ denotes the ordinary partial derivative of the polynomial $a$ with respect to $x_{i}(i=1, \cdots, n)$. Thus $x_{i} D=a_{i}$ and the derivations of $\mathfrak{A}$ are completely determined by the images of the $x_{i}$ and these images may be arbitrarily chosen.

Theorem 2. Let $D$ be a derivation of $\mathfrak{A}$. Then the transformation $\bar{D}$ defined by

$$
\varphi(u) \bar{D}=\phi(u D)
$$

is a derivation of $\mathfrak{B}$ if and only if $\mathfrak{M D} \subseteq \mathfrak{M}$. Moreover, every derivation of $\mathfrak{B}$ is induced in this manner by a derivation of $\mathfrak{A}$.

Proof. Every $\bar{u}$ in $\mathfrak{B}$ is the image under $\phi$ of some $u$ in $\mathfrak{A}$, whence $\bar{D}$ is defined on all of $\mathfrak{B}$. Now assume $\mathfrak{M} D \subseteq \mathfrak{M}$. Suppose $\bar{u}=\phi(u)=\phi(v)$ for elements $u$ and $v$ in $\mathfrak{A}$. Then $u=v+a$ where $a$ is in $\mathfrak{M}, u D=v D+a D$, and $\phi(u D)$ $=\phi(v D)+\phi(a D)$. But $a D$ is in $\mathfrak{M}$, so $\phi(a D)=0, \phi(u D)=\phi(v D)$. Thus $\bar{D}$ is well-defined. Conversely, if $\bar{D}$ is well-defined, then $\phi(u)=\phi(v)$ implies $\phi(u D)$ $=\phi(v D)$. Thus, if $a$ is any element of $\mathfrak{M}$ we have

$$
\phi(u D)=\phi((u+a) D)=\phi(u D)+\phi(a D)
$$

from which it follows that $\phi(a D)=0$ and $a D$ is in $\mathfrak{M}$. We conclude that $\bar{D}$ is well-defined if and only if $\mathfrak{M} D \subseteq \mathfrak{M}$. We will now show that $\bar{D}$ is a derivation of $\mathfrak{B}$.

Let $\bar{u}, \bar{v}$ be elements of $\mathfrak{B}$ and let $\alpha, \beta$ be in $\mathfrak{F}$. Then $\bar{u}=\phi(u), \bar{v}=\phi(v)$ for some $u$ and $v$ in $\mathfrak{A}$, and

$$
\begin{aligned}
(\alpha \bar{u}+\beta \bar{v}) \bar{D} & =[\phi(\alpha u+\beta v)] \bar{D}=\phi((\alpha u+\beta v) D) \\
& =\alpha \phi(u D)+\beta \phi(v D)=\alpha(\bar{u} \bar{D})+\beta(\bar{v} \bar{D}) .
\end{aligned}
$$

Hence $\bar{D}$ is linear. We also have

$$
\begin{aligned}
(\bar{u} \bar{v}) \bar{D} & =[\phi(u v)] \bar{D}=\phi((u v) D) \\
& =\phi((u D) v+u(v D))=(\bar{u} \bar{D}) \bar{v}+\bar{u}(\bar{v} \bar{D})
\end{aligned}
$$

so $\bar{D}$ is a derivation.

Now let $\tilde{D}$ be any derivation of $\mathfrak{B}$. We shall show that $\tilde{D}$ is the induced derivation $\bar{D}$ of some derivation $D$ of $\mathfrak{A}$. Any element $\bar{u}$ of $\mathfrak{B}$ may be written as a polynomial in the generators $\bar{x}_{1}, \cdots, \bar{x}_{n}$. And, as in $\mathfrak{A}, \tilde{D}$ is completely 
determined by its action on the $\bar{x}_{i}$ according to the formula

$$
\bar{u} \tilde{D}=\left(\partial \bar{u} / \partial \bar{x}_{1}\right)\left(\bar{x}_{1} \tilde{D}\right)+\cdots+\left(\partial \bar{u} / \partial \bar{x}_{n}\right)\left(\bar{x}_{n} \tilde{D}\right) .
$$

Choose elements $y_{i}$ in $\mathfrak{A}$ so that $\phi\left(y_{i}\right)=\bar{x}_{i} \widetilde{D}$ for $i=1, \cdots, n$. We can define a derivation $D$ of $\mathfrak{A}$ by specifying that $x_{i} D=y_{i}(i=1, \cdots, n)$. Now let $\bar{D}$ be induced by $D$ according to formula (10). Then $\bar{x}_{i} \bar{D}=\bar{x}_{i} \widetilde{D}$ for $i=1, \cdots, n$. Thus if $\bar{D}$ is a derivation we shall have $\bar{D}=\widetilde{D}$. Therefore it remains only to show that $\mathfrak{M} D \subseteq \mathfrak{M}$.

It is readily seen that if $f=f\left(x_{1}, \cdots, x_{n}\right)$ is any polynomial over $\mathfrak{F}$ in $x_{1}, \cdots, x_{n}$, then $\bar{f}=\phi(f)=f\left(\bar{x}_{1}, \cdots, \bar{x}_{n}\right)$ is the same polynomial with $x_{i}$ replaced by $\bar{x}_{i}$ for $i=1, \cdots, n$. Thus we may write $\partial f / \partial x_{i}=g_{i}\left(x_{1}, \cdots, x_{n}\right)$ and

$$
\phi\left(\partial f / \partial x_{i}\right)=\phi\left(g_{i}\right)=g_{i}\left(\bar{x}_{i}, \cdots, \bar{x}_{n}\right)=\partial \bar{f} / \partial \bar{x}_{i}
$$

for $i=1, \cdots, n$. Now let $u$ be any element of $\mathfrak{M}$. Then $\bar{u}=\phi(u)=0$, and by (9), (11), and (12) we have

$$
\begin{aligned}
\phi(u D) & =\phi\left(\partial u / \partial x_{1}\right) \bar{y}_{1}+\cdots+\phi\left(\partial u / \partial x_{n}\right) \bar{y}_{n} \\
& =\left(\partial \bar{u} / \partial \bar{x}_{1}\right) \bar{y}_{1}+\cdots+\left(\partial \bar{u} / \partial \bar{x}_{n}\right) \bar{y}_{n}=\bar{u} \tilde{D}=0 .
\end{aligned}
$$

Therefore $u D$ is in $\mathfrak{M}$ and the theorem is proved.

We noted earlier that every element of $\mathfrak{A}$ which is not in $\mathfrak{N}$ has an inverse. From this it follows that every proper ideal of $\mathfrak{A}$ is contained in $\mathfrak{R}$. Thus $\mathfrak{M} \subseteq \mathfrak{R}$. Recalling that $\mathfrak{B}$ is $\overline{\mathfrak{D}}$-simple for a set $\overline{\mathfrak{D}}$ of derivations of $\mathfrak{B}$ we now state

THEOREM 3. Let $\mathfrak{D}$ be the set of all derivations $D$ of $\mathfrak{A}$ over $\mathfrak{F}$ such that the induced derivations $\bar{D}$ are in $\overline{\mathfrak{D}}$. Then $\mathfrak{M}$ is a maximal $\mathfrak{D}$-ideal of $\mathfrak{A}$, and an element $u$ of $\mathfrak{A}$ is in $\mathfrak{M}$ if and only if $u$ is in $\mathfrak{N}$ and the elements $u D_{1} \cdots D_{k}$ are in $\mathfrak{R}$ for all values of $k$ and all derivations $D_{i}$ in $\mathfrak{D}$.

Proof. By Theorem 2, if $D$ induces $\bar{D}$ then $\mathfrak{M}$ is a $D$-ideal. Thus $M$ is a $D$-ideal for every $D$ in $\mathfrak{D}$ and hence is a $\mathfrak{D}$-ideal. Let $\mathfrak{R} \neq \mathfrak{A}$ be a $\mathfrak{D}$-ideal properly containing $\mathfrak{M}$ and let $\bar{\Re}=\phi(\Re)$. It is easily verified that $\bar{\Re}$ is a nontrivial $\overline{\mathfrak{D}}$-ideal in $\mathfrak{B}$ contradicting $\overline{\mathfrak{D}}$-simplicity. Hence $\mathfrak{M}$ is a maximal $\mathfrak{D}$-ideal. It is also easily seen that the sum of two $\mathfrak{D}$-ideals is a $\mathfrak{D}$-ideal from which it follows that $\mathfrak{M}$ is maximal in the strong sense that it contains all other $\mathfrak{D}$ ideals.

Now let $u$ be any element of $\mathfrak{M}$. Then $u$ is in $\mathfrak{N}$ and $u D_{1} \cdots D_{k}$ is in $\mathfrak{N}$ for all $k$ and all $D_{i}$ in $\mathfrak{D}$. Conversely, suppose $u$ is in $\mathfrak{N}$ and $u D_{1} \cdots D_{k}$ is in $\mathfrak{N}$ for every product $D_{1} \cdots D_{k}$ of derivations in $\mathfrak{D}$. The ideal generated by $u$ and all $u D_{1} \cdots D_{k}$ is a $\mathfrak{D}$-ideal and hence is contained in $\mathfrak{M}$. Thus $u$ is in $\mathfrak{M}$, and the proof is complete.

To Theorem 3 we have the following immediate 


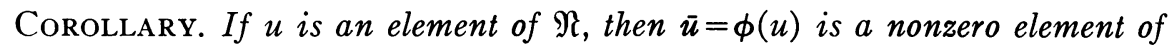
$\mathfrak{B}$ if and only if there is some product $D_{1} \cdots D_{k}$ of derivations $D_{i}$ in $\mathfrak{D}$ such that $u D_{1} \cdots D_{k}$ is nonsingular.

THEOREM 4. Let $u$ be an element of $\mathfrak{N}$ whose terms of degree one are not all zero. Then $u$ is not in $\mathfrak{M}$.

Proof. We assume without loss of generality that $u$ is in $\mathfrak{M}$ and its term of degree one in $x_{1}$ is not zero. Then we may write $u=a x_{1}+v$ where $a$ is nonsingular and $v$ is in $\mathfrak{F}\left[x_{2}, \cdots, x_{n}\right]$. Thus

$$
x_{1}=a^{-1} u-a^{-1} v=u_{0}+v_{0}
$$

where $u_{0}=a^{-1} u$ is in $\mathfrak{M}$ and $v_{0}=-a^{-1} v$ is in the ideal $\mathfrak{B}$ generated by $x_{2}, \cdots, x_{n}$. We observe that every element $f$ of $\mathfrak{B}$ is a polynomial with terms of the form $x_{1}^{r} y$ where $y$ is a monomial in $\mathfrak{F}\left[x_{2}, \cdots, x_{n}\right]$ of degree $t \geqq 1$. If $f$ is not in $\mathfrak{F}\left[x_{2}, \cdots, x_{n}\right]$ we associate with $f$ the number $N(f)$ which is the minimum of the degrees of $y$ for all terms $x_{1}^{r} y$ of $f$ with $r \neq 0$. Note that $1 \leqq N(f) \leqq(n-1)(p-1)$. Now assume it impossible to write $x_{1}=u_{1}+v_{1}$ where $u_{1}$ is in $\mathfrak{M}$ and $v_{1}$ is in $\mathfrak{F}\left[x_{2}, \cdots, x_{n}\right]$. Then we may write $x_{1}=u_{2}+v_{2}$ where $u_{2}$ is in $\mathfrak{M}, v_{2}$ is in $\mathfrak{B}$, and $N\left(v_{2}\right)$ is maximal. Let $x_{1}^{r} y$ be any term of $v_{2}$ with $r \neq 0$ and $y$ of degree $N\left(v_{2}\right)$. By (13) we have for each such $x_{1}^{r} y$

$$
x_{1}^{r} y=x_{1}^{r-1}\left(u_{0}+v_{0}\right) y=x_{1}^{r-1} u_{0} y+x_{1}^{r-1} v_{0} y
$$

where $x_{1}^{r-1} u_{0} y$ is in $\mathfrak{M}$ and $x_{1}^{r-1} v_{0} y$ is a polynomial each term of which has the form $x_{1}^{s} z$ with $z$ in $\mathfrak{F}\left[x_{2}, \cdots, x_{n}\right]$ and the degree of $z$ greater than $N\left(v_{2}\right)$. Hence by means of substitutions as in (14) we may obtain $x_{1}=u_{3}+v_{3}$ with $u_{3}$ in $\mathfrak{M}, v_{3}$ in $\mathfrak{B}$, and $N\left(v_{3}\right)>N\left(v_{2}\right)$. Thus $x_{1}=u_{1}+v_{1}$ with $u_{1}$ in $\mathfrak{M}$ and $v_{1}$ in $\mathfrak{F}\left[x_{2}, \cdots, x_{n}\right]$. But from this it follows that $\bar{x}_{1}=\phi\left(x_{1}\right)=\phi\left(v_{1}\right)$ is in $\mathfrak{F}\left[\bar{x}_{2}, \cdots, \bar{x}_{n}\right]$ contradicting the hypothesis that $\left\{\bar{x}_{1}, \cdots, \bar{x}_{n}\right\}$ is a minimal set of generators of $\mathfrak{B}$. This proves our theorem.

Before we can prove our next theorem we must develop some notation and prove two lemmas on the combinatorial properties of derivations. Let $S=\left\{n_{1}, \cdots, n_{s}\right\}$ be an ordered set of positive integers, the ordering being the natural one. Let $\pi_{1}, \cdots, \pi_{r}$ be ordered subsets of $S$ such that $\pi_{1} \cup \cdots \cup \pi_{r}$ $=S$ and $\pi_{i} \cap \pi_{j}=0$ (the empty set) if $i \neq j$. We shall call the ordered $r$-tuple $\pi=\left(\pi_{1}, \cdots, \pi_{r}\right)$ an $r$-partition of $S$. We now have

Lemma 1. Let $a_{1}, \cdots, a_{r}$ be elements of $\mathfrak{A}$ and let $D_{1}, \cdots, D_{s}$ be derivations in $\mathfrak{D}$. Let

$$
T\left(\pi_{i}\right)=D_{i_{1}} \cdots D_{i_{t}} \quad(i=1, \cdots, r)
$$

if $\pi_{i}=\left\{i_{1}, \cdots, i_{t}\right\}$ is a nonempty ordered subset of the ordered set $S=\{1, \cdots, s\} ;$ and if $\pi_{i}=0$, then $T\left(\pi_{i}\right)$ is to be the identity transformation $I$ of $\mathfrak{A}$. We now assert that 


$$
\left(a_{1} \cdots a_{r}\right) D_{1} \cdots D_{s}=\sum_{\pi}\left[a_{1} T\left(\pi_{1}\right)\right] \cdots\left[a_{r} T\left(\pi_{r}\right)\right]
$$

where $\pi$ ranges over all $r$-partitions of $S$.

Proof. We induce on $s$. If $s=1$, each partition has the form $\pi=(0, \cdots, 0,1,0, \cdots, 0)$ and formula (15) becomes

$$
\left(a_{1} \cdots a_{r}\right) D_{1}=\sum_{i=1}^{r} a_{1} \cdots a_{i-1}\left(a_{i} D_{1}\right) a_{i+1} \cdots a_{r}
$$

which is correct. Now assume (15) correct for $s$ derivations. Then

$$
\begin{aligned}
\left(a_{1} \cdots a_{r}\right) D_{1} \cdots D_{s+1} & =\sum_{\pi}\left\{\left[a_{1} T\left(\pi_{1}\right)\right] \cdots\left[a_{r} T\left(\pi_{r}\right)\right]\right\} D_{s+1} \\
& =\sum_{\pi} \sum_{i}\left[a_{1} T\left(\pi_{1}\right)\right] \cdots\left[a_{i-1} T\left(\pi_{i-1}\right)\right] \\
& \cdot\left[a_{i} T\left(\pi_{i} \cup\{s+1\}\right)\right]\left[a_{i+1} T\left(\pi_{i+1}\right)\right] \cdots\left[a_{r} T\left(\pi_{r}\right)\right] .
\end{aligned}
$$

But if $\pi=\left(\pi_{1}, \cdots, \pi_{r}\right)$ is a general $r$-partition of $\{1, \cdots, s\}$, then $\theta=\left(\pi_{1}, \cdots, \pi_{i-1}, \pi_{i} \cup\{s+1\}, \pi_{i+1}, \cdots, \pi_{r}\right)$ is a general $r$-partition of $\{1, \cdots, s+1\}$. Hence

$$
\left(a_{1} \cdots a_{r}\right) D_{1} \cdots D_{s+1}=\sum_{\theta}\left[a_{1} T\left(\theta_{1}\right)\right] \cdots\left[a_{r} T\left(\theta_{r}\right)\right]
$$

where $\theta=\left(\theta_{1}, \cdots, \theta_{r}\right)$ ranges over all $r$-partitions of $\{1, \cdots, s+1\}$. This is formula (15) for $s+1$ derivations, and the lemma is proved.

We also have

LEMma 2. Let $S_{1}=\left\{i_{1}, \cdots, i_{q}\right\}$ and $S_{2}$ be ordered subsets of the set $S=\{1, \cdots, s\}$ such that $S_{1} \cap S_{2}=0$ and $S_{1} \cup S_{2}=S$, and let $R$ be the set of all $r$-partitions $\pi$ of $S$ with $\pi_{t}=S_{2}$ for some fixed $t$. If $a_{1}, \cdots, a_{r}$ are in $\mathfrak{A}$ and $D_{1}, \cdots, D_{s}$ in $\mathfrak{D}$, then

$$
\begin{aligned}
\sum_{\pi_{i n R} R} & {\left[a_{1} T\left(\pi_{1}\right)\right] \cdots\left[a_{r} T\left(\pi_{r}\right)\right] } \\
& =\left[\left(a_{1} \cdots a_{t-1} a_{t+1} \cdots a_{r}\right) D_{i_{1}} \cdots D_{i_{q}}\right]\left[a_{t} T\left(\pi_{t}\right)\right] .
\end{aligned}
$$

Proof. If $\pi=\left(\pi_{1}, \cdots, \pi_{r}\right)$ with $\pi_{t}=S_{2}$, then

$$
\theta=\left(\pi_{1}, \cdots, \pi_{t-1}, \pi_{t+1}, \cdots, \pi_{r}\right)
$$

is an $(r-1)$-partition of $S_{1}$. Moreover, the correspondence $\pi \leftrightarrow \theta$ is a 1-1 correspondence of $R$ with the set of all $(r-1)$-partitions of $S_{1}$. Our result now follows from Lemma 1 .

We are now able to prove

Theorem 5. The ideal $\mathfrak{M}$ contains no monomial.

Proof. Theorem 4 asserts that $\mathfrak{M}$ contains no monomial of total degree one in $x_{1}, \cdots, x_{n}$. Assume that $\mathfrak{M}$ contains no monomial of degree $r-1$ but 
that $u=x_{1}^{r_{1}} \cdots x_{n}^{r_{n}}$ has degree $r=r_{1}+\cdots+r_{n}$ and is in $\mathfrak{M}$. Then for each $i$ for which $r_{i} \neq 0$ we may write $u=a_{i} x_{i}$ where $a_{i}$ is not in $\mathfrak{M}$. Thus, by the corollary to Theorem 3 , there is a product $G_{i}$ of $t_{i}$ derivations in $\mathfrak{D}$ such that $a_{i} G_{i}$ is nonsingular. We let $i_{0}$ be a value of $i$ for which $t=t_{i}$ is minimal. There is clearly no loss of generality if we assume $i_{0}=1$ and $G=G_{1}=D_{1} \cdots D_{t}$ so that $a_{1} G$ is nonsingular. We now apply $G$ to the element $u$, and by Lemma 1 we obtain

$$
\begin{aligned}
u G & =\left(x_{1}^{r_{1}} \cdots x_{n}^{r_{n}}\right) D_{1} \cdots D_{t} \\
& =\sum_{x}\left[x_{1} T\left(\pi_{11}\right)\right] \cdots\left[x_{1} T\left(\pi_{1 r_{1}}\right)\right] \cdots\left[x_{n} T\left(\pi_{n 1}\right)\right] \cdots\left[x_{n} T\left(\pi_{n r_{n}}\right)\right] .
\end{aligned}
$$

We observe that the constant term of $u G$ is zero since $u$ is in $\mathfrak{M}$ and $u G$ is in $\mathfrak{M}$. Let us now compute the linear term in $x_{1}$ of $u G$. Consider first all summands in (17) with $\pi_{1 j}=0$ for some fixed index $j$. By Lemma 2 the sum of these summands is $\left(a_{1} G\right) x_{1}$ which has a term $\alpha x_{1}$ where $\alpha \neq 0$ is the constant term of the nonsingular element $a_{1} G$. Letting $j=1, \cdots, r_{1}$ we find that the total coefficient of $x_{1}$ from this source is $r_{1} \alpha \neq 0$. Note that any summand in (17) in which $\pi_{i j}=0$ with $i \neq 1$ has $x_{i}$ as a factor and therefore does not have a linear term in $x_{1}$. Thus there remains only the consideration of those summands of (17) in which all $\pi_{i j}$ are nonempty. For such a summand to have a linear term in $x_{1}$ it must be that some $x_{i} T\left(\pi_{i j}\right)$ has a linear term in $x_{1}$ and all other $x_{h} T\left(\pi_{h k}\right)$ are nonsingular. But again it follows from Lemma 2 that the sum of all summands in (17) having $x_{i} T\left(\pi_{i j}\right)$ as a factor is $w=\left(a_{i} H\right)\left[x_{i} T\left(\pi_{i j}\right)\right]$ where $H$ is a product of fewer than $t$ derivations. Hence $a_{i} H$ is singular and $w$ has no linear term. We conclude that $u G$ has a linear term $r_{1} \alpha x_{1}$ contrary to Theorem 4.

We are now essentially through. Albert has shown [2] that for any nonzero element $u$ of $\mathfrak{N}$ there exists an element $v$ of $\mathfrak{A}$ such that $u v=x_{1}^{p-1} \cdots x_{n}^{p-1}$. Thus if $\mathfrak{M} \neq 0$ then $\mathfrak{M}$ contains a monomial, contrary to Theorem 5 . Therefore $\mathfrak{M}=0$ from which Theorem 1 follows.

3 . Some consequences of condition (B). Let $\mathfrak{T}$ be the commutative powerassociative algebra described in $\S 1$. By (1) we see that

$$
\mathfrak{T}=\mathfrak{B}+\mathfrak{R}=\mathfrak{B}+\left(y_{0} \mathfrak{B}, \cdots, y_{m} \mathfrak{B}\right),
$$

and, having determined the structure of $\mathfrak{B}$, we are now in a position to investigate that of $\mathfrak{T}$.

Let $u$ be any element of $\mathfrak{R}$. Then $u=\sum_{j=0}^{n} y_{j} b_{j}$ where $b_{j}$ is in $\mathfrak{B}$. From condition (B) we see that $u=0$ if and only if $\left(y_{i} a\right) u=\sum_{j=0}^{n}\left(y_{i} a\right)\left(y_{j} b_{j}\right)=0$ for all $a$ in $\mathfrak{B}$ and $i=0,1, \cdots, m$. From this it follows that $u=0$ if and only if the relations

$$
\sum_{j=0}^{m}\left(b_{i j} b_{j}-b_{j} D_{i j}\right)=0 \quad(i=0, \cdots, m)
$$




$$
\sum_{j=0}^{m}\left(a D_{i j}\right) b_{j}=0 \quad(i=0, \cdots, m)
$$

hold for every $a$ in $\mathfrak{B}$.

It should be noted that the requirement that the algebra $\mathfrak{T}$ satisfy condition $(B)$ is never inconsistent with the definition of multiplication in $\mathfrak{T}$. The effect of condition (B) is to completely determine the algebra $\mathfrak{T}$ by determining the kernels of the vector space homomorphisms $\mathfrak{B} \rightarrow y_{i} \mathfrak{B}$ for $i=0, \cdots, m$ and the nature of the sum $\mathfrak{R}$. To demonstrate this we let

$$
\mathfrak{T}^{*}=\mathfrak{B}+\mathfrak{R}^{*}=\mathfrak{B}+z_{0} \mathfrak{B}+\cdots+z_{m} \mathfrak{B}
$$

where each vector space $z_{i} \mathfrak{B}$ is an isomorphic copy of $\mathfrak{B}$. Let products in $\mathfrak{T}^{*}$ be defined in terms of the same elements $b_{i j}$ and derivations $D_{i j}$ of $\mathfrak{B}$ which determined products in $\mathfrak{T}$. Since $\mathfrak{T}^{*}$ is a direct sum we see that multiplication is well-defined. Now let $\mathfrak{U}$ be the set of all elements $u$ in $\mathfrak{R}^{*}$ such that $u w=0$ for all $w$ in $\mathfrak{R}^{*}$. The set $\mathfrak{U}$ is an ideal of $\mathfrak{T}^{*}$. The algebra $\mathfrak{T}$ is equivalent to $\mathfrak{T}^{*}-\mathfrak{U}$ and hence exists and is uniquely determined by condition (B) and the choice of the elements $b_{i j}$ and derivations $D_{i j}$ of $\mathfrak{B}$.

4. A special case with $m=2$. In this section we shall construct a class of examples of the algebras $\mathfrak{T}$ in which $\mathfrak{R}=\left(y_{0} \mathfrak{B}, \cdots, y_{m} \mathfrak{B}\right)$ with $m=2$ and $\mathfrak{R}$ is not a direct sum. We let $\mathfrak{B}=\mathfrak{F}[e, x, y], \mathfrak{T}=\mathfrak{B}+\left(y_{0} \mathfrak{B}, y_{1} \mathfrak{B}, y_{2} \mathfrak{B}\right)$ and let

$$
\begin{array}{ll}
x D_{01}=e, & y D_{01}=x^{p-1}, \\
x D_{02}=x^{2} y, & y D_{02}=x y, \\
x D_{12}=-x, & y D_{12}=x y^{2}, \\
b_{11}=0, \quad b_{12}=e, \quad b_{22}=-x^{2} .
\end{array}
$$

The algebra $\mathfrak{B}$ is $D_{01}$-simple [2] and hence is $\left\{D_{i j}\right\}$-simple. Thus $\mathfrak{T}$ satisfies condition (A). We complete the definition of $\mathfrak{T}$ by imposing condition (B). As a routine consequence of formulas (18) and (19) we now have Lemma 3 which we state without proof.

Lемма 3. In this special case an element $y_{0} b_{0}+y_{1} b_{1}+y_{2} b_{2}$ of $\mathbb{R}$ is zero if and only if $b_{0}=-b_{2} x, b_{1}=0$, and $b_{2}=x^{p-1} f(y)+y^{p-1} g(x)$ where $f(y)$ and $g(x)$ are polynomials over $\mathfrak{F}$ in $y$ and $x$ respectively.

It follows from Lemma 3 that $\mathfrak{T}$ is not a direct sum. In fact we may write

$$
\mathfrak{T}=\mathfrak{B}+\left(y_{0} \mathfrak{B}+y_{1} \mathfrak{B}, y_{2} \mathfrak{B}\right)
$$

and we see that $\left(y_{0} \mathfrak{B}+y_{1} \mathfrak{B}\right) \cap y_{2} \mathfrak{B}$ is spanned by the independent vectors $y_{2} x^{p-1} y^{j}$ and $y_{2} x^{i} y^{p-1}$ where $i=0, \cdots, p-1$ and $j=0, \cdots, p-2$. Hence $\mathfrak{T}$ has dimension $4 p^{2}-2 p+1$. We will show next that $\mathfrak{I}$ not only fails to be a direct sum as presently represented, but, furthermore, Albert's construction cannot yield a representation of $\mathfrak{T}$ as a direct sum.

Let $\overline{\mathfrak{B}}=\mathfrak{F}\left[\bar{e}, \bar{z}_{1}, \cdots, \bar{z}_{r}\right]=\bar{e} \mathfrak{F}+\overline{\mathfrak{R}}$ be a polynomial algebra over $\mathfrak{F}$ with 
unity $\bar{e}$ and generators $\bar{z}_{1}, \cdots, \bar{z}_{r}$ such that $\bar{z}_{i}^{p}=0$ for $i=1, \cdots, r$ but which are otherwise independent. Suppose there exist $x_{0}, \cdots, x_{m}$ such that $\mathfrak{T}=\overline{\mathfrak{B}}+\overline{\mathfrak{Q}}$ where $\overline{\mathfrak{Q}}=x_{0} \overline{\mathfrak{B}}+\cdots+x_{m} \overline{\mathfrak{B}}$ is a direct sum. We will denote by $\bar{a}, \bar{b}$, etc. elements of $\overline{\mathfrak{B}}$ and by $\bar{b}_{i j}$ and $\bar{D}_{i j}$ the elements and derivations of $\overline{\mathfrak{B}}$ which define multiplication in this new representation of $\mathfrak{T}$. We observe that $\bar{e}=e$ since $\overline{\mathfrak{B}}$ and $\mathfrak{B}$ have the same unity element as $\mathfrak{T}$. We may write expressions for the $x_{k} e$ in terms of the original representation of $\mathfrak{T}$. Thus

$$
x_{k} e=a_{k}+y_{0} b_{k}+y_{1} c_{k}+y_{2} d_{k}
$$

where $a_{k}, b_{k}, c_{k}, d_{k}$ are in $\mathfrak{B}$ and $k=0, \cdots, m$. Since $\mathfrak{T}$ is power-associative and $p$ is an odd prime we have

$$
\left(x_{k} b\right)^{p}=\left[\left(x^{k} \bar{b}\right)^{2}\right]^{(p-1) / 2}\left(x_{k} \bar{b}\right)=x_{k}\left(\bar{b}_{k k}^{(p-1) / 2} b^{p}\right)
$$

for $k=0, \cdots, m$, and similarly

$$
\left(y_{k} b\right)^{p}=y_{k}\left(b_{k k}^{(p-1) / 2} b^{p}\right)
$$

for $k=0,1,2$. From (23), (25), (26), (27) and Lemma 3 we see that

$$
x_{0} e=\left(x_{0} e\right)^{p}=a_{0}^{p}+y_{0} b_{0}^{p}=\alpha+y_{0} \beta
$$

where $\alpha$ and $\beta$ are in $\mathfrak{F}$. Since $\left(x_{0} e\right)^{2}=e$ it follows that $\alpha=0$ and $\beta= \pm 1$. Thus $x_{0} e= \pm y_{0} e$ and we can now prove

Lemma 4. The algebras $\mathfrak{B}$ and $\overline{\mathfrak{B}}$ coincide as do the spaces $\mathfrak{R}$ and $\overline{\mathfrak{R}}$.

Proof. Since $x_{0} e= \pm y_{0} e$ and $\left(x_{0} e\right)\left(x_{k} e\right)=0$ for $k=1, \cdots, m$, we see by (25) that $a_{k}=0$ for $k=0, \cdots, m$. Hence $\overline{\mathfrak{R}} \subseteq \mathfrak{R}$. Since $\overline{\mathfrak{B}}$ is $\left\{\bar{D}_{i j}\right\}$-simple there is a derivation $\bar{D}_{s t}$ of $\overline{\mathfrak{B}}$ such that $\overline{\mathfrak{R}}$ is not a $\bar{D}_{s t}$-ideal. From this it follows that each of $\overline{\mathfrak{B}}, x_{s} \overline{\mathfrak{B}}$, and $x_{t} \overline{\mathfrak{B}}$ has dimension $p^{r}$. Thus $3 p^{r} \leqq 4 p^{2}-2 p+1$ which implies $r \leqq 2$. If $r<2$ the dimension of $\overline{\mathfrak{R}}$ is seen to be greater than that of $\mathfrak{R}$. Thus $r=2$ and $\overline{\mathfrak{l}}=\mathfrak{l}$.

We have shown that $\mathfrak{B}+\mathfrak{R}=\overline{\mathfrak{B}}+\mathfrak{R}$. Now let $b$ be any element of $\mathfrak{B}$. Then $b=\bar{b}+u$ for elements $\bar{b}$ in $\overline{\mathfrak{B}}$ and $u$ in $\mathfrak{R}$. Let $w$ be an arbitrary element of $\mathbb{R}$. We see that $u w=b w-\bar{b} w$ is in $\mathfrak{B}$ and in $\&$. Thus $u w=0$ for all $w$ in $\mathfrak{R}$. Therefore $u=0$, and the lemma is proved.

We now have $\overline{\mathfrak{B}}=\mathfrak{B}=\mathfrak{F}[e, x, y]$ and

$$
\mathfrak{T}=\mathfrak{B}+\left(y_{0} \mathfrak{B}+y_{1} \mathfrak{B}, y_{2} \mathfrak{B}\right)=\mathfrak{B}+x_{0} \mathfrak{B}+\cdots+x_{m} \mathfrak{B} .
$$

We shall show that this leads to a contradiction. Setting $a_{k}=0(k=0, \cdots, m)$ in (25) we obtain

$$
x_{k} e=y_{0} b_{k}+y_{1} c_{k}+y_{2} d_{k} \quad(k=0, \cdots, m) .
$$

Leмma 5. If $\mathfrak{T}=\mathfrak{B}+x_{0} \mathfrak{B}+\cdots+x_{m} \mathfrak{B}$ is a direct sum, then $m=2$ and there exist elements $v$ and $w$ in $\mathfrak{B}$ such that $b_{2}=-d_{2} x+x y v$ and $c_{2}=x y w$. 
Proof. The dimension of $x_{0} \mathfrak{B}$ is always the same as that of $\mathfrak{B}$, and we noted earlier that this must also be true for at least one other $x_{k} \mathfrak{B}$. Hence we may assume that $x_{1} \mathfrak{B}$ has dimension $p^{2}$. It follows that $x_{k} \mathfrak{B}$ has dimension less than $p^{2}$ for $k=2, \cdots, m$. Hence if $U=x^{p-1} y^{p-1}$, then $x_{k} U=0$ for $k \geqq 2$, and we see from (28) and Lemma 3 that $b_{k}$ and $c_{k}$ are in $\mathfrak{N}$ for $k \geqq 2$. We see also that $x_{1} U \mp x_{0} b_{1} U=y_{1} c_{1} U \neq 0$ since $x_{0} e= \pm y_{0} e$ and $x_{0} \mathfrak{B}+x_{1} \mathfrak{B}$ is a direct sum. Hence $c_{1}$ is not in $\mathfrak{R}$.

Now let $u_{0}, \cdots, u_{m}$ be elements of $\mathfrak{B}$ such that $y_{2} e=x_{0} u_{0}+\cdots+x_{m} u_{m}$. From (28) and Lemma 3 we see that $c_{1} u_{1}+\cdots+c_{m} u_{m}=0$, and, since $c_{1}$ is not in $\mathfrak{N}$ but $c_{2}, \cdots, c_{m}$ are in $\mathfrak{N}$, this implies that $u_{1}$ is in $\mathfrak{N}$. Now, also by Lemma $3, d_{1} u_{1}+\cdots+d_{m} u_{m}-e$ is in $\mathfrak{N}$ and hence $d_{k}$ is not in $\mathfrak{N}$ for some $k \geqq 2$. Without loss of generality we assume $d_{2}$ is not in $\mathfrak{R}$.

Let $\mathbb{E}$ be the subspace of $\mathfrak{B}$ consisting of all elements $u$ such that $x_{2} u=0$. Since $d_{2}$ is nonsingular, it follows from (28) and Lemma 3 that $u=x^{p-1} F(y)$ $+y^{p-1} G(x)$ for some polynomials $F(y)$ and $G(x)$. Let $s$ be the dimension of $₹$. Then $x_{2} \mathfrak{B}$ has dimension $p^{2}-s$, and we see that $s \geqq 2 p-1$. Thus $x_{2} u=0$ for all possible choices of the polynomials $F(y)$ and $G(x)$. Thus $x_{2} \mathfrak{B}$ has dimension $p^{2}-2 p+1$ and $m=2$.

We have shown that the space $\&$ consists of all $u$ in $\mathfrak{B}$ of the form $u=x^{p-1} F(y)+y^{p-1} G(x)$. From Lemma 3 we see that $c_{2} u=0$ and $\left(b_{2}+d_{2} x\right) u=0$ for all $u$ in $\$$. It therefore follows that $c_{2}=x y w$ and $b_{2}=-d_{2} x+x y v$ for some $v$ and $w$ in $\mathfrak{B}$. We can now obtain our main result which we state as

TheOREM 6. The algebra $\mathfrak{T}$ cannot be represented as a direct sum.

Proof. We compute $\bar{b}_{02}$ and single out those terms which possibly give rise to a linear term in $x$ alone. We recall that $\bar{b}_{02}=0$ by (2). Using (20), $\cdots$, (23) and Lemma 5 we see that

$$
\begin{aligned}
\bar{b}_{02} & =\left(x_{0} e\right)\left(x_{2} e\right)= \pm\left(y_{0} e\right)\left(y_{0} b_{2}+y_{1} c_{2}+y_{2} d_{2}\right) \\
& = \pm\left(b_{2}-c_{2} D_{01}-d_{2} D_{02}\right)=\mp d_{2} x+\Omega
\end{aligned}
$$

where $\Omega$ is a sum of terms each having $x^{2}$ or $y$ as a factor. Since $d_{2}$ is nonsingular $b_{02} \neq 0$. This contradiction proves the theorem.

\section{REFERENCES}

1. A. A. Albert, $A$ theory of power-associative commutative algebras, Trans. Amer. Math. Soc. vol. 69 (1950) pp. 503-527.

2. - On commutative power-associative algebras of degree two, Trans. Amer. Math. Soc. vol. 74 (1953) pp. 323-343.

3. - On partially stable algebras, Trans. Amer. Math. Soc. vol. 84 (1957) pp. 430-443.

4. Nathan Jacobson, Classes of restricted Lie algebras of characteristic p. II, Duke Math. J. vol. 10 (1943) pp. 107-121.

UNIVERSITY OF MiNNESOTA, MinNeAPOLIS, Minnesota 